\title{
POINTWISE CONVERGENCE ALONG RESTRICTED DIRECTIONS FOR THE FRACTIONAL SCHRÖDINGER EQUATION
}

\author{
SHOBU SHIRAKI
}

\begin{abstract}
We consider the pointwise convergence problem for the solution of Schrödinger-type equations along directions determined by a given compact subset of the real line. This problem contains Carleson's problem as the most simple case and was studied in general by Cho-Lee-Vargas. We extend their result from the case of the classical Schrödinger equation to a class of equations which includes the fractional Schrödinger equations. To achieve this, we significantly simplify their proof by completely avoiding a time localization argument.
\end{abstract}

\section{INTRODUCTION}

Let $d \geq 1, a>1$ and consider the fractional Schrödinger equation

$$
\begin{cases}\partial_{t} u(x, t)=i\left(-\Delta_{x}\right)^{\frac{a}{2}} u(x, t) & (x, t) \in \mathbb{R}^{d} \times \mathbb{R} \\ u(x, 0)=f(x) & x \in \mathbb{R}^{d} .\end{cases}
$$

It is well-known that for a sufficiently nice initial data $f$, the solution can be written as

$$
u(x, t)=e^{i t(-\Delta)^{\frac{a}{2}}} f(x):=\left(\frac{1}{2 \pi}\right)^{d} \int_{\mathbb{R}^{d}} e^{i\left(x \cdot \xi+t|\xi|^{a}\right)} \widehat{f}(\xi) d \xi,
$$

where $\widehat{f}(\xi):=\int_{\mathbb{R}^{d}} e^{-i x \cdot \xi} f(x) d x$. When $a=2$, this is the standard Schrödinger equation from quantum mechanics. The general case has arisen in recent years in physical models and has been the subject of numerous papers (see, for example, [6, 7, 11, 12, 13, 15]). Associated with the fractional Schrödinger equation, it is natural to try to determine the minimum level of regularity $s$ which guarantees that the limit

$$
\lim _{\substack{(y, t) \rightarrow(x, 0) \\(y, t) \in \Gamma_{x}}} e^{i t(-\Delta)^{\frac{a}{2}}} f(y)=f(x) \quad \text { a.e. }
$$

holds whenever $f \in H^{s}(\mathbb{R})$. Here, $H^{s}\left(\mathbb{R}^{d}\right)$ is the Sobolev space of order $s$ whose norm is given by

$$
\|f\|_{H^{s}\left(\mathbb{R}^{d}\right)}=\left\|(1-\Delta)^{\frac{s}{2}} f\right\|_{L^{2}\left(\mathbb{R}^{d}\right)}
$$

and $\Gamma_{x} \subset \mathbb{R}^{d} \times[-1,1]$ is a convergence domain correspondings to each $x \in \mathbb{R}^{d}$.

The classical case, known widely as Carleson's problem, is concerned with the case of vertical lines $\Gamma_{x}=\{x\} \times[-1,1]$. Here, when $d=1$ it is known that (1) holds if and only if $s \geq \frac{1}{4}$; see the work of Carleson [3] and Dahlberg-Kenig [8] for the case $a=2$, and also see the work of Sjölin [18, for $a>1$. The higher dimensional case $d \geq 2$ has been subject to a recent flurry of activity. When $a=2$, Bourgain [2] showed that $s \geq \frac{1}{2}-\frac{1}{2(d+1)}$ is necessary for (1) for $d \geq 2$, and Du-Guth-Li [9] and Du-Zhang [10] have shown $s>\frac{1}{2}-\frac{1}{2(d+1)}$ is sufficient for (1) for $d=2$ and $d \geq 3$, respectively (for important earlier contributions see, for example, papers by

Date: March 7, 2019. 
Vega [20, Lee [14] and Bourgain [1]). In addition, for $a>1$, Cho-Ko [4] proved that (1) holds if $s>\frac{1}{2}-\frac{1}{2(d+1)}$ and $d \geq 2$.

Non-tangential convergence corresponds to the case

$$
\Gamma_{x}=\{x+t \theta: t \in[-1,1] \text { and } \theta \in \mathbb{B}\},
$$

where $\mathbb{B} \subset \mathbb{R}^{d}$ is a given euclidean ball which is centered at the origin, that is, $\Gamma_{x}$ is a conical region with vertex at $(x, 0)$ and aperture determined by the radius of $\mathbb{B}$. In this case, it is known that (1) holds if and only if $s>\frac{d}{2}$. The sufficiency part of this claim follows easily by a well-known argument using Sobolev embedding and the delicate necessity part has proved by Sjögren-Sjölin in [17].

When $d=1$, the classical case and the non-tangential case were unified in a natural way by Cho-Lee-Vargas [5] who proved that (1) holds in the case

$$
\Gamma_{x}=\{x+t \theta: t \in[-1,1] \text { and } \theta \in \Theta\}
$$

when $a=2$ and $s>\frac{\beta(\Theta)+1}{4}$. Here, $\Theta \subset \mathbb{R}$ is a given compact set and $\beta(\Theta)$ denotes the upper Minkowski dimension of $\Theta$. Our main goal in this paper is to improve the result in [5] by extending to a class of equations which includes the fractional Schrödinger equation for $a>1$. We define the evolution operator $S_{t}$ on appropriate input functions by

$$
S_{t} f(x)=\frac{1}{2 \pi} \int_{\mathbb{R}} e^{i(x \xi+t \Phi(\xi))} \widehat{f}(\xi) d \xi .
$$

Here, $\Phi: \mathbb{R} \rightarrow \mathbb{R}$ is a $C^{2}$ function which satisfies for some $C_{1}>0$,

$$
|\xi|\left|\Phi^{\prime \prime}(\xi)\right| \geq C_{1}
$$

for all $|\xi| \geq 1$. Moreover, for some $C_{2}>0$,

$$
|\xi|\left|\Phi^{\prime \prime}(\xi)\right| \geq C_{2}\left|\Phi^{\prime}(\xi)\right|
$$

for all $|\xi| \geq 1$. It is trivial to verify that $\Phi(\xi)=|\xi|^{a}$ satisfies these conditions when $a>1$.

Our main result is the following.

Theorem 1. Let $\Theta \subset \mathbb{R}$ be compact and suppose $\Phi \in C^{2}(\mathbb{R})$ satisfies $(2)$ and (3). For any $q \in[1,4]$ and $s>\frac{\beta(\Theta)+1}{4}$, there exists a constant $C_{q, s}$ such that

$$
\left\|\sup _{(t, \theta) \in[-1,1] \times \Theta}\left|S_{t} f(\cdot+t \theta)\right|\right\|_{L^{q}(-1,1)} \leq C_{q, s}\|f\|_{H^{s}(\mathbb{R})} .
$$

By standard arguments, we thus obtain the associated pointwise convergence.

Corollary 2. Let $\Theta \subset \mathbb{R}$ be compact and suppose $\Phi \in C^{2}(\mathbb{R})$ satisfies (2) and (3). If $s>\frac{\beta(\Theta)+1}{4}$, then

$$
\lim _{\substack{(y, t) \rightarrow(x, 0) \\ y-x \in t \Theta}} S_{t} f(y)=f(x) \quad \text { a.e. }
$$

whenever $f \in H^{s}(\mathbb{R})$.

Theorem 1 improves the result in [5] in two respects; the class of evolution operators has been widened from the case $\Phi(\xi)=|\xi|^{2}$ to those satisfying (2) and (3), and our maximal estimates are valid for $q \in[1,4]$ (the estimate in $[5$, was proved in the only case $q=2$ ). While the proof in [5] may be modified in a straightforward way to go beyond the classical case $\Phi(\xi)=|\xi|^{2}$ to a certain extent, it seems to us to be difficult to handle case $\Phi(\xi)=|\xi|^{a}$ with $a$ close to 1 . Indeed, the argument in [5] rests on a certain widely used time localization argument which becomes increasingly weak as $a$ approaches 1 . To overcome this significant obstacle, we remove the use of the time localization lemma; this simplification to the proof 
has allowed us to handle the case $\Phi(\xi)=|\xi|^{a}$ for any $a>1$. Further explanation of this point will follow our proof of Theorem 1 in Section 3 . Prior to that, we prepare for the proof of Theorem 1 in Section 2.

\section{Preliminaries}

Notations. Associated with the operator $S_{t}$ given above by

$$
S_{t} f(x)=\frac{1}{2 \pi} \int_{\mathbb{R}} e^{i(x \xi+t \Phi(\xi))} \widehat{f}(\xi) d \xi
$$

and a fixed compact set $\Theta \subset \mathbb{R}$, we define the maximal operator $M_{\Theta}$ by

$$
M_{\Theta} f(x)=\sup \left\{\left|S_{t} f(x+t \theta)\right|:-1 \leq t \leq 1, \theta \in \Theta\right\} .
$$

Also, we recall that the upper Minkowski dimension of $\Theta$ is defined by

$$
\beta(\Theta)=\inf \left\{r>0: \limsup _{\delta \rightarrow 0} N(\Theta, \delta) \delta^{r}=0\right\},
$$

where $N(\Theta, \delta)$ denotes the smallest number of $\delta$-intervals which cover $\Theta$.

We will use the following notations frequently:

- $I=(-1,1)$.

- $q^{\prime}=\frac{q}{q-1}$ : Hölder conjugate of $q \in[1, \infty]$.

- $A \lesssim B: A \leq C B$ for some constant $C>0$.

- $A \gtrsim B: A \geq C B$ for some constant $C>0$.

- $A \sim B: C^{-1} B \leq A \leq C B$ for some constant $C>0$.

- $L_{x}^{p} L_{t}^{q} L_{\theta}^{r}$ : The Lebesgue space with norm

$$
\|F\|_{L_{x}^{p} L_{t}^{q} L_{\theta}^{r}}=\left(\int\left(\int\left(\int|F(x, t, \theta)|^{r} d \theta\right)^{\frac{q}{r}} d t\right)^{\frac{p}{q}} d x\right)^{\frac{1}{p}}
$$

where the domains of integration will be clear from the context.

Useful lemmas. The following lemmas will be crucial for the oscillatory integral estimates in the proof of Theorem 1. Applying these lemmas appropriately essentially allows us to avoid the time localization lemma, which is used in [5].

Lemma 3 (van der Corput's lemma). Suppose $\lambda>1$ and we have $\left|\phi^{(k)}(x)\right| \geq 1$ for all $(a, b)$. If $k=1$ and $\phi^{\prime}$ is monotonic on $(a, b)$, or simply $k \geq 2$, then there exists a constant $C_{k}$ such that

$$
\left|\int_{a}^{b} e^{i \lambda \phi(x)} \psi(x) d x\right|<C_{k} \lambda^{-\frac{1}{k}}\left(\int_{a}^{b}\left|\psi^{\prime}(x)\right| d x+\|\psi\|_{L^{\infty}}\right) .
$$

For a proof of van der Corput's lemma, we refer the reader to [19].

Lemma 4. Let $1 \leq q \leq 4$. There exists a constant $C_{q} \geq 0$ such that

$$
\left|\iiint \int g(x, t) h\left(x^{\prime}, t^{\prime}\right)\right| x-\left.x^{\prime}\right|^{-\frac{1}{2}} d x d x^{\prime} d t d t^{\prime} \mid \leq C_{q}\|g\|_{L_{x}^{q^{\prime} L_{t}^{1}}}\|h\|_{L_{x}^{q^{\prime}} L_{t}^{1}}
$$

where the integrals are taken over $(x, t),\left(x^{\prime}, t^{\prime}\right) \in I \times[-1,1]$.

Proof. Denoting $G(x)=\|g(x, \cdot)\|_{L^{1}}$ and $H\left(x^{\prime}\right)=\left\|h\left(x^{\prime}, \cdot\right)\right\|_{L^{1}}$,

$$
\left|\iiint \int g(x, t) h\left(x^{\prime}, t^{\prime}\right)\right| x-\left.x^{\prime}\right|^{-\frac{1}{2}} d x d x^{\prime} d t d t^{\prime}\left|\leq \int_{-1}^{1} \int_{-1}^{1} G(x) H(y)\right| x-\left.x^{\prime}\right|^{-\frac{1}{2}} d x d x^{\prime} .
$$


By the Hardy-Littlewood-Sobolev inequality,

$$
\begin{aligned}
\int_{-1}^{1} \int_{-1}^{1} G(x) H\left(x^{\prime}\right)\left|x-x^{\prime}\right|^{-\frac{1}{2}} d x d x^{\prime} & \lesssim\|G\|_{L^{\frac{4}{3}}(I)}\|H\|_{L^{\frac{4}{3}}(I)} \\
& \lesssim\|g\|_{L_{x}^{q^{\prime}} L_{t}^{1}}\|h\|_{L_{x}^{q^{\prime}} L_{t}^{1}}
\end{aligned}
$$

where the last inequality is obtained by Hölder's inequality since $\frac{4}{3} \leq q^{\prime}$ from our assumption.

\section{Proof of Theorem 1}

Proof of Theorem 1. We fix $q \in[2,4]$ and without loss of generality, we suppose $\Theta \subset[-1,1]$. The case $q \in[1,2)$ follows immediately by Hölder's inequality.

The first half of the proof is based on the proof in [5]. Suppose $\psi_{0} \in C_{0}^{\infty}(I)$ and $\psi \in C_{0}^{\infty}\left(\left(-2,-\frac{1}{2}\right) \cup\left(\frac{1}{2}, 2\right)\right)$ give rise to a standard dyadic partition of unity

$$
\psi_{0}(\xi)+\sum_{k \geq 1} \psi_{k} \equiv 1
$$

where $\psi_{k}=\psi\left(\frac{\cdot}{2^{k-1}}\right)$. For each $0 \leq k \in \mathbb{Z}$, the frequency localization operator $P_{k}$ is defined by

$$
\widehat{P_{k} f}(\xi)=\psi_{k}(\xi) \widehat{f}(\xi) \text {. }
$$

Then,

$$
\left\|M_{\Theta} f\right\|_{L^{q}(I)}^{q} \lesssim\left\|M_{\Theta} P_{0} f\right\|_{L^{q}(I)}^{q}+\sum_{k \geq 1}\left\|M_{\Theta} P_{k} f\right\|_{L^{q}(I)}^{q}
$$

The first term is relatively easy to estimate. In fact,

$$
\begin{aligned}
\left\|M_{\Theta} P_{0} f\right\|_{L^{q}(I)}^{q} & \lesssim \int_{\mathbb{R}} \psi_{0}(\xi)|\widehat{f}(\xi)| d \xi \\
& \lesssim\|f\|_{L^{2}} \\
& \lesssim\|f\|_{H^{s}}
\end{aligned}
$$

for $s \geq 0$ and thereby this case is no problem.

For the remaining cases, fix a parameter $\sigma$ (to be chosen at the end of the proof) satisfying

$$
\frac{q}{4} \leq \sigma \leq 1
$$

Since $\Theta$ is compact, for each $\lambda>0$ there exists a finite collection $\left\{\Omega_{j}(\lambda)\right\}_{j=1}^{N}$ which satisfies

$$
\Theta \subset \bigcup_{j=1}^{N} \Omega_{j}(\lambda)
$$

where $\left|\Omega_{j}(\lambda)\right| \leq \lambda^{-\sigma}$ for each $j$ and $N=N\left(\Theta, \lambda^{-\sigma}\right)$ is the smallest number of $\lambda^{-\sigma}$-intervals which cover $\Theta$. For fixed $k$ and $x \in(-1,1)$,

$$
M_{\Theta} P_{k} f(x)^{q} \leq \sum_{j=1}^{N} \sup _{\substack{1 \leq t \leq 1 \\ \theta \in \Omega \\ \theta, j}}\left|S_{t} P_{k} f(x+t \theta)\right|^{q},
$$

where $\Omega_{k, j}=\Omega_{j}\left(2^{k}\right)$, and therefore

$$
\sum_{k \geq 1}\left\|M_{\Theta} P_{k} f\right\|_{L^{q}(I)}^{q} \leq \sum_{k \geq 1} \sum_{j=1}^{N}\left\|M_{\Omega_{k, j}} P_{k} f\right\|_{L^{q}(I)}^{q} .
$$

Now, we shall introduce the following useful proposition. 
Proposition 5. Let $k \geq 1$ and $\Omega$ be an interval with $|\Omega| \leq 2^{-\sigma k}$. Then, there exists a constant $C_{q}>0$ such that

$$
\left\|M_{\Omega} P_{k} f\right\|_{L^{q}(I)} \leq C_{q} 2^{\frac{k}{4}}\|f\|_{L^{2}}
$$

holds for all $f \in L^{2}(\mathbb{R})$.

Proof of Proposition 5. Set $\lambda=2^{k}$ and

$$
T f(x, t, \theta):=\chi(x, t, \theta) \int_{\mathbb{R}} e^{i((x+t \theta) \xi+t \Phi(\xi))} f(\xi) \psi\left(\frac{\xi}{\lambda}\right) d \xi,
$$

where $\chi=\chi_{I \times[-1,1] \times \Omega}$. Then (7) follows from

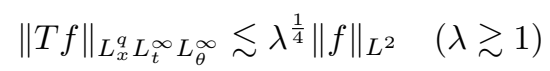

since

$$
\begin{aligned}
\left\|M_{\Omega} P_{k} f\right\|_{L^{q}(I)} & \sim\|T \widehat{f}\|_{L_{x}^{2} L_{t}^{\infty} L_{\theta}^{\infty}} \\
& \lesssim \lambda^{\frac{1}{4}}\|\widehat{f}\|_{L^{2}} \\
& \lesssim \lambda^{\frac{1}{4}}\|f\|_{L^{2}}
\end{aligned}
$$

by Plancherel's theorem. Let us consider the dual form of (8), which is

$$
\left\|T^{*} F\right\|_{L^{2}} \lesssim \lambda^{\frac{1}{4}}\|F\|_{L_{x}^{q^{\prime}} L_{t}^{1} L_{\theta}^{1}}
$$

where

$$
T^{*} F(\xi)=\psi\left(\frac{\xi}{\lambda}\right) \iiint \chi\left(x^{\prime}, t^{\prime}, \theta^{\prime}\right) e^{-i\left(\left(x^{\prime}+t^{\prime} \theta^{\prime}\right) \xi+t^{\prime} \Phi(\xi)\right)} F\left(x^{\prime}, t^{\prime}, \theta^{\prime}\right) d x^{\prime} d t^{\prime} d \theta^{\prime} .
$$

Then,

$$
\begin{aligned}
\| & T^{*} F \|_{L^{2}}^{2} \\
= & \lambda \int \psi^{2}(\xi) \iiint \iiint \chi(x, t, \theta) \chi\left(x^{\prime}, t^{\prime}, \theta^{\prime}\right) \\
& \times e^{i\left(\lambda\left(x-x^{\prime}+t \theta+t^{\prime} \theta^{\prime}\right) \xi+\left(t-t^{\prime}\right) \Phi(\lambda \xi)\right)} \bar{F}(x, t, \theta) F\left(x^{\prime}, t^{\prime}, \theta^{\prime}\right) d x d t d \theta d x^{\prime} d t^{\prime} d \theta^{\prime} d \xi \\
= & \lambda \int_{W} \int_{W^{\prime}} \chi(w) \chi\left(w^{\prime}\right) \bar{F}(w) F\left(w^{\prime}\right) K_{\lambda}\left(w, w^{\prime}\right) d w d w^{\prime} \\
= & \sum_{\ell=1}^{3} \lambda \iint_{V_{\ell}} \chi(w) \chi\left(w^{\prime}\right) \bar{F}(w) F\left(w^{\prime}\right) K_{\lambda}\left(w, w^{\prime}\right) d w d w^{\prime} \\
= & : A_{1}+A_{2}+A_{3} .
\end{aligned}
$$

Here, we denote $w=(x, t, \theta) \in W$ and $w^{\prime}=\left(x^{\prime}, t^{\prime}, \theta^{\prime}\right) \in W$, where $W:=I \times$ $[-1,1] \times \Omega$. Also,

$$
\begin{gathered}
K_{\lambda}\left(w, w^{\prime}\right)=\int_{\mathbb{R}} e^{i \phi(\lambda \xi)} \psi^{2}(\xi) d \xi \\
\phi\left(\xi, w, w^{\prime}\right)=\left(x-x^{\prime}+t \theta-t^{\prime} \theta^{\prime}\right) \xi+\left(t-t^{\prime}\right) \Phi(\xi),
\end{gathered}
$$

and

$$
\left\{\begin{array}{l}
V_{1}=\left\{\left(w, w^{\prime}\right) \in W \times W:\left|x-x^{\prime}\right|<4\left|t-t^{\prime}\right|\right\} \\
V_{2}=\left\{\left(w, w^{\prime}\right) \in W \times W:\left|x-x^{\prime}\right| \geq 4\left|t-t^{\prime}\right| \text { and }\left|x-x^{\prime}\right| \geq 4 \lambda^{-\sigma}\right\} \\
V_{3}=\left\{\left(w, w^{\prime}\right) \in W \times W:\left|x-x^{\prime}\right| \geq 4\left|t-t^{\prime}\right| \text { and }\left|x-x^{\prime}\right|<4 \lambda^{-\sigma}\right\} .
\end{array}\right.
$$

Thus, (9) follows from

for each $\ell=1,2,3$.

$$
A_{\ell} \lesssim \lambda^{\frac{1}{2}}\|F\|_{L_{x}^{q^{\prime}} L_{t}^{1} L_{\theta}^{1}}^{2}
$$


The term $A_{1}$. Let us start with an estimate of $A_{1}$. Since

$$
\left|\phi^{\prime \prime}(\lambda \xi)\right|=\lambda^{2}\left|t-t^{\prime}\right|\left|\Phi^{\prime \prime}(\lambda \xi)\right| \gtrsim \lambda\left|x-x^{\prime}\right|
$$

holds from (2), we are allowed to apply Lemma 3 to get

$$
\left|K_{\lambda}\left(x, x^{\prime}, t, t^{\prime}, \theta, \theta^{\prime}\right)\right| \lesssim\left(\lambda\left|x-x^{\prime}\right|\right)^{-\frac{1}{2}} .
$$

By using Lemma 4, it follows that

$$
\begin{aligned}
A_{1} & \leq \lambda^{\frac{1}{2}} \iint_{V_{1}} \chi\left(w^{\prime}\right)\left|F\left(w^{\prime}\right)\right| \chi(w)|\bar{F}(w)|\left|x-x^{\prime}\right|^{-\frac{1}{2}} d w d w^{\prime} \\
& \lesssim \lambda^{\frac{1}{2}}\|F\|_{L_{x}^{q^{\prime}} L_{t}^{1} L_{\theta}^{1}}^{2} .
\end{aligned}
$$

The term $A_{2}$. Next, we shall consider $A_{2}$. In this case, the following key relationship holds:

$$
\left|x-x^{\prime}+t \theta-t^{\prime} \theta^{\prime}\right| \sim\left|x-x^{\prime}\right| .
$$

Indeed,

$$
\begin{aligned}
\left|x-x^{\prime}+t \theta-t^{\prime} \theta^{\prime}\right| & \geq\left|x-x^{\prime}\right|-\left|t-t^{\prime}\right|-\left|\theta-\theta^{\prime}\right| \\
& \geq \frac{3}{4}\left|x-x^{\prime}\right|-\lambda^{-\sigma} \\
& \geq \frac{1}{2}\left|x-x^{\prime}\right| .
\end{aligned}
$$

Similarly, the other way holds, too.

Now, let us observe that for all $\left(w, w^{\prime}\right) \in V_{2}$, we have

$$
\left|K_{\lambda}\left(w, w^{\prime}\right)\right| \lesssim \lambda\left|x-x^{\prime}\right|^{-\frac{1}{2}} .
$$

Before proving (11), we note that

$$
A_{2} \lesssim \lambda^{\frac{1}{2}}\|F\|_{L_{x}^{q^{\prime}} L_{t}^{1} L_{\theta}^{1}}^{2}
$$

immediately follows by using Lemma 4 as before.

To see (11), let us split $K_{\lambda}$ into $B_{1}$ and $B_{2}$ as follows

$$
\begin{aligned}
K_{\lambda}\left(w, w^{\prime}\right) & =\int_{U_{1}} e^{i \phi(\lambda \xi)} \psi^{2}(\xi) d \xi+\int_{U_{2}} e^{i \phi(\lambda \xi)} \psi^{2}(\xi) d \xi \\
& =: B_{1}+B_{2}
\end{aligned}
$$

where

$$
U_{1}=\left\{\xi \in \mathbb{R}:\left|x-x^{\prime}+t \theta-t^{\prime} \theta^{\prime}\right| \geq 2\left|t-t^{\prime}\right|\left|\Phi^{\prime}(\lambda \xi)\right|\right\}
$$

and

$$
U_{2}=\left\{\xi \in \mathbb{R}:\left|x-x^{\prime}+t \theta-t^{\prime} \theta^{\prime}\right|<2\left|t-t^{\prime}\right|\left|\Phi^{\prime}(\lambda \xi)\right|\right\} .
$$

For $B_{1}$, we have

$$
\begin{aligned}
\left|\phi^{\prime}(\lambda \xi)\right| & \geq \lambda\left|x-x^{\prime}+t \theta-t^{\prime} \theta^{\prime}\right|-\lambda\left|t-t^{\prime}\right|\left|\Phi^{\prime}(\lambda \xi)\right| \\
& \geq \frac{\lambda}{2}\left|x-x^{\prime}+t \theta-t^{\prime} \theta^{\prime}\right| \\
& \geq \frac{\lambda}{4}\left|x-x^{\prime}\right| \\
& >\lambda^{1-\sigma} \\
& \geq 1
\end{aligned}
$$


because of (6). From (2) and the intermediate value theorem, $\Phi^{\prime \prime}(\xi)$ is single-signed on $(-\infty,-1]$ and $[1, \infty)$, which guarantees that $\Phi^{\prime}(\xi)$ is monotone for $|\xi| \geq 1$. Hence, $U_{1}$ consists of at most three intervals. Invoking Lemma 3

$$
\begin{aligned}
B_{1} & \lesssim\left(\lambda\left|x-x^{\prime}\right|\right)^{-1} \\
& \lesssim\left(\lambda\left|x-x^{\prime}\right|\right)^{-\frac{1}{2}} .
\end{aligned}
$$

On the other hand, for $B_{2}$, it follows from (3) that

$$
\begin{aligned}
\left|\phi^{\prime \prime}(\lambda \xi)\right| & =\lambda^{2}\left|t-t^{\prime}\right|\left|\Phi^{\prime \prime}(\lambda \xi)\right| \\
& \gtrsim \lambda\left|t-t^{\prime}\right|\left|\Phi^{\prime}(\lambda \xi)\right| \\
& \gtrsim \lambda\left|x-x^{\prime}+t \theta-t \theta^{\prime}\right| \\
& \gtrsim \lambda\left|x-x^{\prime}\right| .
\end{aligned}
$$

Then, by using Lemma 3 , we obtain

$$
B_{2} \lesssim\left(\lambda\left|x-x^{\prime}\right|\right)^{-\frac{1}{2}}
$$

Therefore, (11) holds.

The term $A_{3}$. It remains to show

$$
A_{3} \lesssim \lambda^{\frac{1}{2}}\|F\|_{L_{x}^{q^{\prime}} L_{t}^{1} L_{\theta}^{1}}^{2}
$$

Trivially,

$$
\left|K_{\lambda}\left(w, w^{\prime}\right)\right| \lesssim 1
$$

so by the dual form of Young's convolution inequality

$$
\begin{aligned}
& \int_{-1}^{1} \int_{-1}^{1}\|F(x, \cdot, \cdot)\|_{L_{t}^{1} L_{\theta}^{1}}\left\|F\left(x^{\prime}, \cdot, \cdot\right)\right\|_{L_{t}^{1} L_{\theta}^{1}} \chi_{\left[-4 \lambda^{-\sigma}, 4 \lambda^{-\sigma}\right]}\left(x-x^{\prime}\right) d x d x^{\prime} \\
& \lesssim\|F\|_{L_{x}^{q^{\prime}} L_{t}^{1} L_{\theta}^{1}}^{2}\left\|\chi_{\left[-4 \lambda^{-\sigma}, 4 \lambda^{-\sigma}\right]}\right\|_{L^{\frac{q}{2}}} \\
& \sim \lambda^{1-\frac{2 \sigma}{q}\|F\|_{L_{x}^{q^{\prime}} L_{t}^{1} L_{\theta}^{1}}^{2}}
\end{aligned}
$$

Therefore, we conclude that

$$
A_{3} \lesssim \lambda^{1-\frac{2 \sigma}{q}}\|F\|_{L_{x}^{q^{\prime}} L_{t}^{1} L_{\theta}^{1}}^{2} \lesssim \lambda^{\frac{1}{2}}\|F\|_{L_{x}^{q^{\prime}} L_{t}^{1} L_{\theta}^{1}}^{2}
$$

whenever (6), as claimed.

When $\lambda$ is large, by the definition of the upper Minkowski dimension, for small $\varepsilon>0$ there is a constant $C_{\varepsilon}>0$ depending on $\varepsilon$ such that

$$
N\left(\Theta, \lambda^{-\sigma}\right) \leq C_{\varepsilon} \lambda^{\sigma \beta(\Theta)+\varepsilon} .
$$


Thus, if we also let $\widehat{\tilde{P}_{k} f}=\tilde{\psi}_{k} \widehat{f}$, where $\tilde{\psi} \in C_{0}^{\infty}\left(\left(-4,-\frac{1}{4}\right) \cup\left(\frac{1}{4}, 4\right)\right)$ with $\tilde{\psi} \equiv 1$ on $\left(-2,-\frac{1}{2}\right) \cup\left(\frac{1}{2}, 2\right)$, then

$$
\begin{aligned}
\sum_{k \geq 1} \sum_{j=1}^{N}\left\|M_{\Omega_{k, j}} P_{k} f\right\|_{L^{q}(I)}^{q} & =\sum_{k \geq 1} \sum_{j=1}^{N}\left\|M_{\Omega_{k, j}} P_{k} \tilde{P}_{k} f\right\|_{L^{q}(I)}^{q} \\
& \lesssim \sum_{k \geq 1} \sum_{j=1}^{N} 2^{\frac{q k}{4}}\left\|\tilde{P}_{k} f\right\|_{L^{2}}^{q} \\
& \lesssim \sum_{k \geq 1} 2^{k q\left(\frac{\sigma}{q} \beta(\Theta)+\frac{1}{4}+\frac{\sigma}{q} \varepsilon\right)}\left\|\tilde{P}_{k} f\right\|_{L^{2}}^{q} \\
& \sim \sum_{k \geq 1} 2^{-\frac{\sigma}{q} \varepsilon}\left(\int_{\operatorname{supp} \tilde{\psi_{k}}} 2^{2 k\left(\frac{\sigma \beta(\Theta)}{q}+\frac{1}{4}+\frac{2 \sigma \varepsilon}{q}\right)}|\widehat{f}(\xi)|^{2} d \xi\right)^{\frac{q}{2}} \\
& \lesssim\|f\|_{H}^{\frac{\sigma \beta(\Theta)}{q}+\frac{1}{4}+\frac{2 \sigma \varepsilon}{q}} \cdot
\end{aligned}
$$

Therefore, for arbitrary $\varepsilon>0$,

$$
\left\|M_{\Theta} f\right\|_{L^{q}(I)} \lesssim\|f\|_{H} \frac{\sigma \beta(\Theta)}{q}+\frac{1}{4}+\varepsilon
$$

holds. Referring to $(6)$, we shall let the Sobolev order be as small as possible to conclude that for arbitrary $\varepsilon>0$

$$
\left\|M_{\Theta} f\right\|_{L^{q}(I)} \lesssim\|f\|_{H} \frac{\beta(\Theta)+1}{4}+\varepsilon,
$$

which ends the proof.

Remarks. The critical step in the above proof of Theorem 1 is Proposition 5 . The corresponding result in [5] (Lemma 3.1), stated for $q=2$ and $\Phi(\xi)=|\xi|^{2}$, is established through the following steps: $T T^{*}$ argument, the time localization lemma, Schur's lemma and then an oscillatory integral argument. Following this approach, one may extend by simple modification, for example, to the case $\Phi(\xi)=|\xi|^{a}$ with $a \geq \frac{3}{2}$. The time localization lemma reduces to the case of time intervals of length $\lambda^{1-a}$, and for $a$ close to 1 this causes certain technical difficulties in the estimation of the oscillatory integrals which arise; in particular, the relationship 10 breaks down if we follow their argument as it stands. In order to overcome the significant technical difficulty, we removed the use of the time localization lemma and replaced this with appropriate decompositions of the domain $W \times W$.

Acknowledgment. The author would like to thank Neal Bez for his encouragement and a lot of discussions.

\section{REFERENCES}

[1] J. Bourgain, On the Schrödinger maximal function in higher dimension, Tr. Mat. Inst. Steklova 280 (2013), 46-60.

[2] J. Bourgain, A note on the Schrödinger maximal function, J. Anal. Math. 130 (2016), 393396.

[3] L. Carleson, Some analytic problems related to statistical mechanics. In: Euclidean harmonic analysis (Proc. Sem., Univ. Maryland, College Park, Md., 1979), Lecture Notes in Math. 779 (1980), 5-45.

[4] C. H. Cho, H. Ko, A note on maximal estimates of generalized Schrödinger equation, arXiv: 1809.03246.

[5] C. H. Cho, S. Lee, A. Vargas, Problems on pointwise convergence of solutions to the Schrödinger equation, J. Fourier Anal. Appl. 18 (2012), 972-994.

[6] Y. Cho, S. Lee, Strichartz estimates in spherical coordinates, Indiana Univ. Math. J. 62 (2013), 991-1020.

[7] P. Constantin, J.-C. Saut, Local smoothing properties of dispersive equations, J. Amer. Math. Soc. 1 (1988), 413-439. 
[8] B. E. J. Dahlberg, C. E. Kenig, A note on the almost everywhere behavior of solutions to the Schrödinger equation. In: Harmonic analysis (Minneapolis, Minn., 1981), Lecture Notes in Math. 908 (1982), 205-209.

[9] X. Du, L. Guth, X. Li, A sharp Schrödinger maximal estimate in $\mathbb{R}^{2}$, Ann. of Math. 186 (2017), 607-640.

[10] X. Du, R. Zhang, Sharp $L^{2}$ estimate of Schrödinger maximal function in higher dimensions, Ann. of Math., to appear.

[11] B. Guo, Z. Huo, Global well-posedness for the fractional nonlinear Schrödinger equation, Comm. Partial Differential Equations 36 (2011), 247-255.

[12] Z. Guo, Y. Wang, Improved Strichartz estimates for a class of dispersive equations in the radial case and their applications to nonlinear Schrödinger and wave equations, J. Anal. Math. 124 (2014), 1-38.

[13] N. Laskin, Fractional quantum mechanics and Lévy path integrals, Phys. Lett. A 268 (2000), 298-305.

[14] S. Lee, On pointwise convergence of the solutions to Schrödinger equations in $\mathbb{R}^{2}$, Int. Math. Res. Not. (2006), 1-21.

[15] B. Pausader, The cubic fourth-order Schrödinger equation, J. Funct. Anal. 256 (2009), 24732517.

[16] K. M. Rogers, P. Villarroya, Sharp estimates for maximal operators associated to the wave equation, Ark. Mat. 46 (2008), 143-151.

[17] P. Sjögren, P. Sjölin, Convergence properties for the time dependent Schrödinger equation, Ann. Acad. Sci. Fenn. 14 (1989), 13-25.

[18] P. Sjölin, Regularity of solutions to the Schrödinger equation. Duke Math. J. 55 (1987), 699-715.

[19] E. M. Stein, Harmonic Analysis, Princeton University Press, 329-374 (1994).

[20] L. Vega, Schrödinger equations: pointwise convergence to the initial data, Proc. Amer. Math. Soc. 102 (1988), 874-878.

(Shobu Shiraki) Department of Mathematics, Graduate school of Science and EngiNEERING, SAitama University, SAitama, 338-8570, JAPAN

E-mail address: s.shiraki.446@ms.saitama-u.ac.jp 